ThE Astrophysical JournaL, 465:79-90, 1996 July 1

(C) 1996. The American Astronomical Society. All rights reserved. Printed in U.S.A.

\title{
DETECTION OF THE TIP OF THE RED GIANT BRANCH IN NGC $5128^{1}$
}

\author{
Roberto Soria, ${ }^{2}$ Jeremy R. Mould,${ }^{2}$ Alan M. Watson,${ }^{3}$ John S. Gallagher III ${ }^{3}$ Gilda E. Ballester, ${ }^{4}$ \\ Christopher J. Burrows, ${ }^{5}$ Stefano Casertano, ${ }^{6}$ John T. Clarke, ${ }^{4}$ David Crisp,${ }^{7}$ Richard E. Griffiths, ${ }^{6}$ \\ J. Jeff Hester, ${ }^{8}$ John G. Hoessel, ${ }^{2}$ Jon A. Holtzman, ${ }^{9}$ Paul A. Scowen, ${ }^{8}$ \\ KARl R. Stapelfeldt, ${ }^{7}$ John T. Trauger, ${ }^{7}$ AND JAMEs A. WestPhal ${ }^{10}$ \\ Received 1995 October 30; accepted 1996 January 5
}

\begin{abstract}
We present a color-magnitude diagram of more than 10,000 stars in the halo of the galaxy NGC 5128 (Centaurus A), based on WFPC2 images through $V$-band and $I$-band filters. The position of the red giant branch (RGB) stars is compared with the loci of the red giant branch in six well-studied globular clusters and in the dwarf elliptical galaxy NGC 185; the tip of the RGB is signalled by an observed turn-up in the luminosity function at $I \simeq 24.1 \pm 0.1 \mathrm{mag}$; this yields a distance modulus $(m-M)_{0}=27.8$ \pm 0.2 for NGC 5128 (i.e., a distance of $3.6 \pm 0.2 \mathrm{Mpc}$ ), in agreement with previous determinations based on the planetary nebulae luminosity function and on the surface brightness fluctuations technique.

The presence of an intermediate-age stellar population $(\sim 5 \mathrm{Gyr})$ is suggested by the luminosity function of the asymptotic giant branch stars, extending up to $I=22.6 \mathrm{mag}$ (for $V-I>2$ ) and $M_{\mathrm{bol}} \sim-5$ mag; however, the number of these stars constrains the intermediate-age stellar population in the halo of NGC 5128 to be less than $\sim 10 \%$ of the total. The color distribution at constant $I$ magnitude, albeit affected by the completeness level of our sample, strongly suggests a mean value of $[\mathrm{Fe} / \mathrm{H}]>-0.9 \mathrm{dex}$, possibly similar to the value found in M31 and higher than that observed in NGC 185. Like the M31 halo, the halo of NGC 5128 exhibits a broad range of levels of chemical enrichment.

Subject headings: galaxies: distances and redshifts - galaxies: individual (NGC 5128) galaxies: stellar content - stars: Hertzsprung-Russell diagram
\end{abstract}

\section{INTRODUCTION}

Understanding the nature of elliptical galaxies is one of the major ripe problems of astrophysics. Baade's surmise that the stellar population of elliptical galaxies was equivalent to the stellar population of the halo of the Milky Way was based upon resolution of the dwarf elliptical galaxies of the Local Group. More recent insights have suggested galaxy mergers as the origin of these pressure-supported stellar systems (Toomre \& Toomre 1972; Schweizer 1994). Either way, the characteristics of the stellar population remain the proving ground for theories of the origin of these galaxies.

NGC 5128 is classified "S0 pec"; its linkage to elliptical galaxies is through its dominant spheroid (Graham 1979); its linkage to the lenticular class is through its striking and gratuitous disk (Quillen, Graham, \& Frogel 1993). Merger scenarios have been explored by Malin, Quinn, \& Graham (1983). There is a neutral hydrogen remnant in the halo of

\footnotetext{
${ }^{1}$ Based on observations with the NASA/ESA Hubble Space Telescope.

${ }^{2}$ Mount Stromlo and Siding Spring Observatories, Institute of Advanced Studies, Australian National University, Private Bag, Weston Creek Post Office, ACT 2611, Australia.

${ }^{3}$ Department of Astronomy, University of Wisconsin-Madison, 475 North Charter Street, Madison, WI 53706.

${ }_{4}$ Department of Atmospheric and Oceanic Sciences, University of Michigan, 2455 Hayward, Ann Arbor, MI 48109.

${ }_{5}^{5}$ Space Telescope Science Institute, 3700 San Martin Drive, Baltimore, MD 21218.

${ }^{6}$ Department of Astronomy, Johns Hopkins University, 3400 North Charles Street, Baltimore, MD 21218

7 Jet Propulsion Laboratory, 4800 Oak Grove Drive, Mail Stop 179225, Pasadena, CA 91109.

${ }^{8}$ Department of Physics and Astronomy, Arizona State University, Tyler Mall, Tempe, AZ 85287.

${ }_{9}^{9}$ Lowell Observatory, Mars Hill Road, Flagstaff, AZ 86001.

${ }^{10}$ Division of Geological and Planetary Sciences, California Institute of Technology, Pasadena, CA 91125.
}

the galaxy (Schiminovich et al. 1993). The galaxy's activity provides a laboratory for gas/jet interaction (Sutherland, Bicknell, \& Dopita 1993). As Centaurus A, it is the nearest major active galactic nucleus, a factor of 5 closer than M87. Its proximity makes it a useful testing ground for early-type galaxy distance indicators (see, e.g., Hui et al. 1993; de Vaucouleurs 1993).

The resolution and sensitivity of WFPC2 on the Hubble Space Telescope (HST) provide a unique opportunity to probe the stellar population of this nearest large early-type system. Until now the Local Group spheroids have been the only systems that could be resolved into individual stars. We begin the study by examining a halo field some $9 \mathrm{kpc}$ south of the center of the galaxy; the position of our field is illustrated in Figure 1 (image taken from the STScI Digitized Sky Survey). We find a stellar population that is in a close relation to that seen in the dwarf ellipticals previously examined from the ground (Mould, Kristian, \& Da Costa 1983, 1984) and to the halo of M31 (Mould \& Kristian 1986).

\section{OBSERVATIONS AND PHOTOMETRY}

Eight CCD images of a $160^{\prime \prime} \times 160^{\prime \prime}$ field in the halo of NGC 5128 were obtained on 1995 May 30 with WFPC2: four through the F555W filter $(1100,1100,500$, and $500 \mathrm{~s}$ exposures), and the other four through F814W $(600,600$, 1300 , and $1300 \mathrm{~s}$ exposures). Figure 1 shows the geometry of the high-resolution planetary camera chip and the three low-resolution wide field chips superposed on the galaxy. A full description of WFPC2 can be found in both Trauger et al. (1994) and the instrument handbook (Burrows 1994). Bias subtraction, flat-fielding, and cosmic-ray removal were performed according to the prescriptions of Holtzman et al. (1995b) and of Stetson (1995); the two series of four images were then averaged in order to obtain a single image 


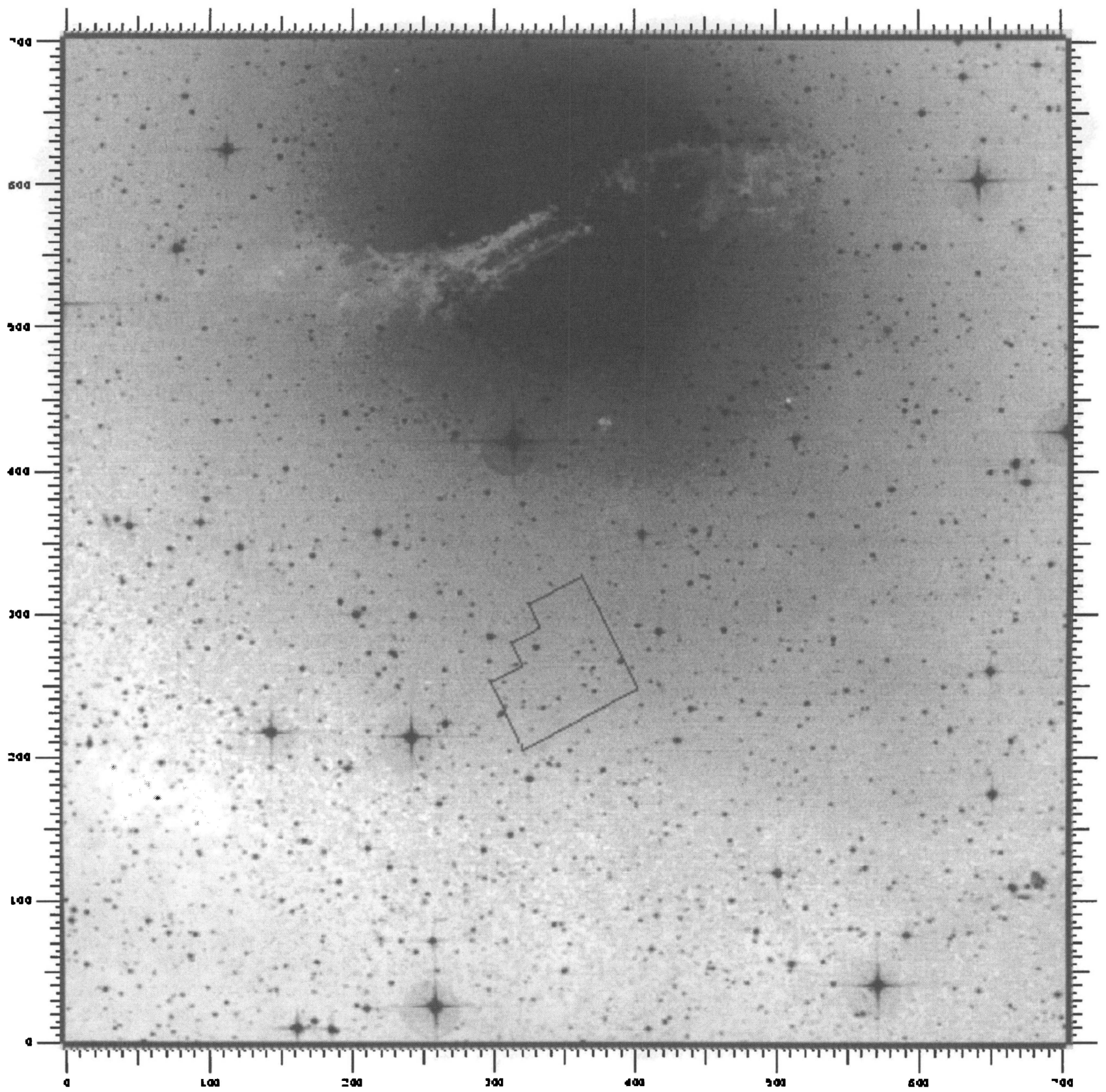

FIG. 1.-Position of our field in the halo of NGC 5128; image from the STScI Digitized Sky Survey; size: $20^{\prime} \times 20^{\prime}$

(divided into four chips and rescaled to $1000 \mathrm{~s}$ ) for each filter. A section of the resulting F814W image from chip 2 in the Wide Field Camera has been printed in Figure 2 (size: $\left.40^{\prime \prime} \times 40^{\prime \prime}\right)$. We then vignetted the four frames and applied a correction to the pixel area accounting for geometric distortion (the pixel area was rescaled to the average one, following Stetson's convention).

A preliminary list of stars detected in each chip for each filter was compiled using the automatic star-finding algorithm in DAOPHOT; their instrumental magnitudes were determined with the reduction packages ALLSTAR and ALLFRAME (Stetson 1991a, b, 1994, 1995) that fitted a point-spread function (psf) to each star. The matching program DAOMASTER allowed us to discard stars detected in only one color, as well as stars whose position in the F555W image did not match their position in the
F814W image to within 0"04. Eventually, some 11,000 stars were retained (563 of them detected in chip 1, 3422 in chip 2, 3273 in chip 3, and 3827 in chip 4). We then chose several (relatively) isolated bright stars in each chip in order to compare the values of the magnitudes estimated by the point-spread function-fitting algorithm in ALLFRAME with those obtained with aperture photometry, using an aperture radius $=0.5$, corresponding to 5 pixels in chips 2 , 3 , and 4 (Wide Field Camera) and to 11 pixels in chip 1 (Planetary Camera). Unfortunately, the lack of bright stars in chip 1 made our calibration rather uncertain for the PC (we estimated an error of a few tenths of a magnitude); therefore, we decided to adopt Stetson's (1995) zero points for all four chips, for the sake of consistency. We also introduced a linear correction for the charge transfer efficiency problems explained in Holtzman et al. (1995a): stars located 


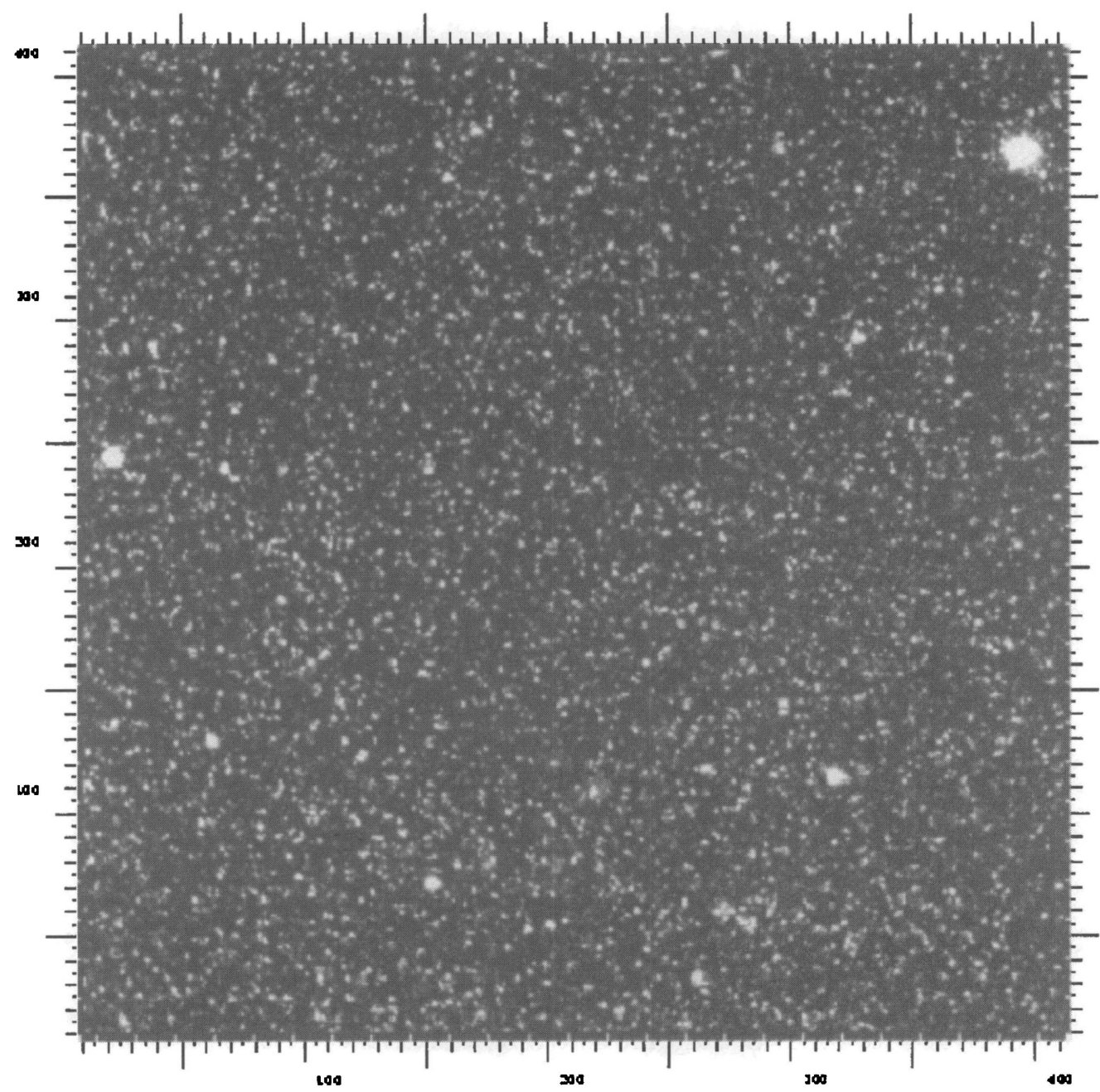

FIG. 2.-Image of a $40^{\prime \prime} \times 40^{\prime \prime}$ field in the halo of NGC 5128 taken with WF2 of the WFPC2, through the infrared filter F814W; the image has already been bias- and cosmic-ray-subtracted.

in the 800th row were adjusted to be 0.04 mag brighter, while stars in the first row were left unchanged. ${ }^{11}$

Finally, the conversion from the WFPC2 flight photometric system to the standard Cousins colors was implemented using the coefficients tabulated in Holtzman et al. (1995a): F555W is roughly equivalent to $V$, and F814W to $I$ (see also Burrows 1994). In detail, we used the following relation between ALLFRAME psf magnitudes $v$ and $i$, and the standard magnitudes $V$ and $I$ :

$$
\left\{\begin{array}{l}
V=v+2.5 \log \left(t_{\text {exp }}\right)-A-B_{V}(V-I)-C_{V}(V-I)^{2} \\
I=i+2.5 \log \left(t_{\text {exp }}\right)-A-B_{I}(V-I)-C_{I}(V-I)^{2}
\end{array},\right.
$$

where $B_{V}=-0.052, C_{V}=0.027, B_{I}=-0.063, C_{I}=0.025$, and the values of $A$ for the different filters and chips have

TABLE 1

Value of the Adopted Zero PoINT $A$ For the DiFFERENT FILTERS AND CHIPS

\begin{tabular}{ccccc}
\hline \hline Filter & PC1 & WF2 & WF3 & WF4 \\
\hline$V \ldots \ldots$ & $0.967 \pm 0.018$ & $0.958 \pm 0.009$ & $0.950 \pm 0.009$ & $0.973 \pm 0.011$ \\
$I \ldots \ldots$ & $1.861 \pm 0.017$ & $1.823 \pm 0.014$ & $1.842 \pm 0.009$ & $1.870 \pm 0.013$
\end{tabular}

\footnotetext{
${ }^{11}$ Although this is a standard correction, it is unclear whether it is required for data with this illumination level. We regard our magnitudes as uncertain by $+0 /-0.02$ from this unknown.
}

been listed in Table 1; $A$ is the sum of DAOPHOT photometric offset $(=+25)$, minus the zero point (z.p.) for a given filter (z.p. $=21.724$ for $V$; z.p. $=20.840$ for $I$ ), minus a gain factor (different for each chip but $\simeq 0.75 \mathrm{mag}$ for $7 e^{-}$gain), minus the term required to correct the instrumental magnitudes to a 0.5 aperture radius (of order $\sim 0.1$ ), minus a rescaling factor $=2.5 \log 4$ (all pixel values had been divided by four, initially, as explained in Holtzman et al. 1995a).

\section{COMPLETENESS}

Figures 3 and 4 display the $(I, V-I)$ and the $(V, V-I)$ color-magnitude diagrams of some 10,000 stars in our field. First, as explained below in this section, we evaluated the photometric errors: we found that the estimates calculated by ALLFRAME are not reliable (they tend to underestimate the true error); therefore, we used the standard technique of adding "artificial" stars in our frames, with random but a priori known position and brightness, and we reran all the star-finding algorithms on the new images. We then compared the magnitude of each added star as determined by ALLFRAME with the value given as input. Not all the fake stars could be recovered, and this allowed us to plot lines in both color-magnitudes diagrams signalling the approximate $50 \%$ completeness levels (see Figs. 9 and 10).

The ADDSTAR task of DAOPHOT proved to be a powerful tool for estimating the completeness of our sample 


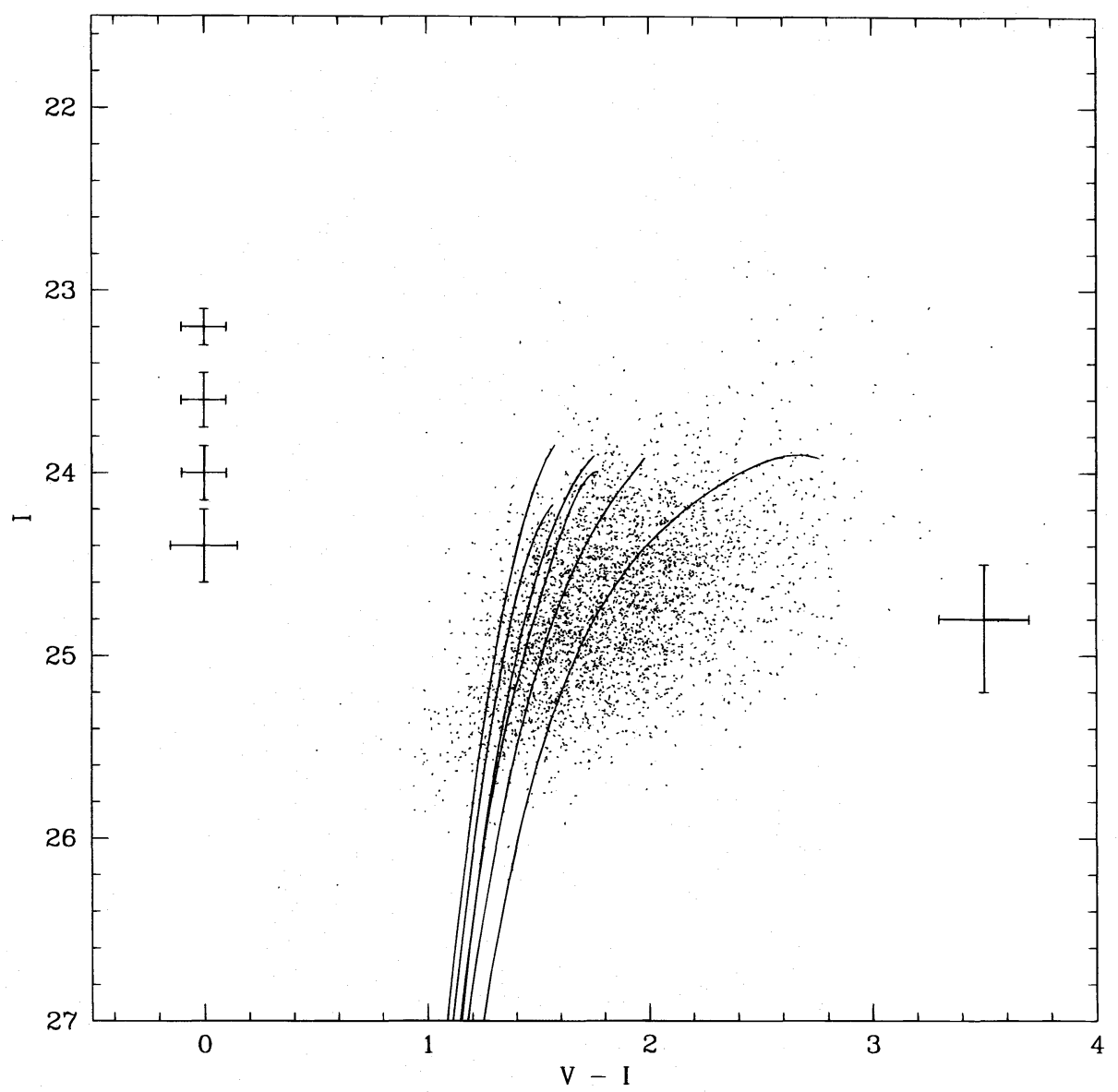

Fig. 3.-Color-magnitude diagram in the $(I, V-I)$ plane for about 10,000 stars in the halo of NGC 5128. The mean photometric error at various magnitudes has been determined by using the ADDSTAR task of DAOPHOT. The solid curves are the loci of the giant branches in six globular clusters studied by Da Costa \& Armandroff (1990); from left to right, they are M15, NGC 6397, M2, NGC 6752, NGC 1851, and 47 Tuc; see the text for the adopted values of reddening and modulus.

and the photometric error as a function of magnitude. We added 500 stars on each of the WFPC 2 chips, both in the $V$ and in the $I$ images, with random position (but coincident in both filters) and random magnitudes in the range $22.5<I<27.5 \mathrm{mag}$ and $23.5<V<28.5 \mathrm{mag}$ (it is easy to verify that the completeness level is 1 for stars brighter than $I=22.5 \mathrm{mag}$ and $V=23.5$ and is 0 for stars fainter than $I=27.5 \mathrm{mag}$ and $V=28.5$ ). The new frames containing all the real plus the fake stars were then run through the same routines (DAOPHOT, ALLSTAR, ALLFRAME, DAOMASTER) in order to produce a new list of stars and respective magnitudes; this was then compared with the original list of artificial stars (from which we subtracted all the stars falling in the vignetted areas of each image). The ratio between the initial and the final number of added stars allowed us to determine the completeness functions at each magnitude, as shown in Figure 5; we applied these curves when we determined the color (hence also the metallicity) distribution at fixed values of $I$ (see $\S 6$ ). A limitation of the ADDSTAR task is the fact that stars are created with the same point-spread function that is then used as a template to detect them; in other words, they are probably slightly easier to find than real stars, and the completeness level may be a little overestimated.

The artificial stars experiment also helped us determine the photometric errors in $V, I$, and $V-I$ as a function of magnitude; the original magnitudes were compared with the PSF magnitudes derived by ALLFRAME, and the resulting error bars are shown in Figures 3 and 4. Systematic errors begin to affect the data near or below the $50 \%$ completeness level, where only stars with positive fluctuations in their luminosity are detected. Occasionally, errors larger than 1 mag occur because of an overlapping of stars in our crowded field; this explains only in part the large number of stars found above the TRGB; besides, this effect is more evident in the $I$ magnitude, and such blended stars are more frequently found at very red colors.

\section{COMPARISON WITH GLOBULAR CLUSTER GIANT BRANCHES}

In the $(I, V-I)$ plane, it is easy to see that the dominant contribution comes from the red giant branch (RGB) stars (cf. the analogous CMDs for the dwarf elliptical galaxy NGC 185 plotted in Lee, Freedman, \& Madore 1993a). The number density of the RGB stars rises sharply at $I \approx 24$ mag for $V-I \gtrsim 1.5 \mathrm{mag}$, which probably defines the tip of the red giant branch. The RGB tip in the C-M diagram represents the locus of the core helium flash for stars with electron-degenerate cores; stars will follow the RGB during their post-main-sequence evolution, up to the TRGB, where we expect to find a discontinuity in the luminosity function. After the helium flash, stars will at first fall onto the horizontal branch and then move up quickly along the asymptotic giant branch (AGB). Distinguishing between red giant stars near the TRGB and asymptotic giant stars may not always be easy, but the timescale for the evolution along 


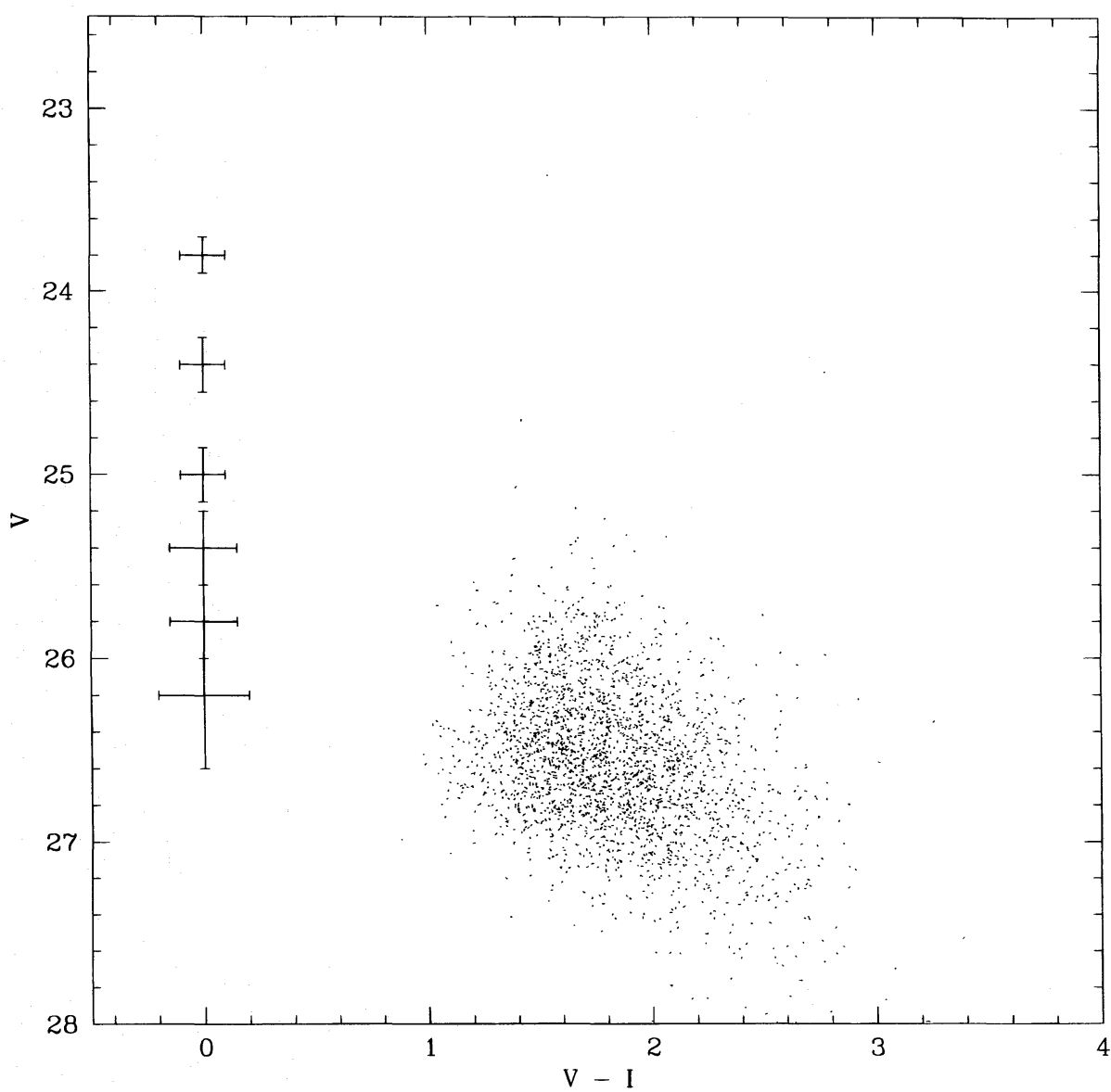

FIG. 4.-Color-magnitude diagram in the $(V, V-I)$ plane for the same stars as in Fig. 3. The photometric error has been determined as before.

the AGB is much shorter than the time spent on the RGB; hence, the number of AGB stars observed is correspondingly lower. AGB stars will give the most significant contribution to the stars lying about 1 mag above the RGB tip, as

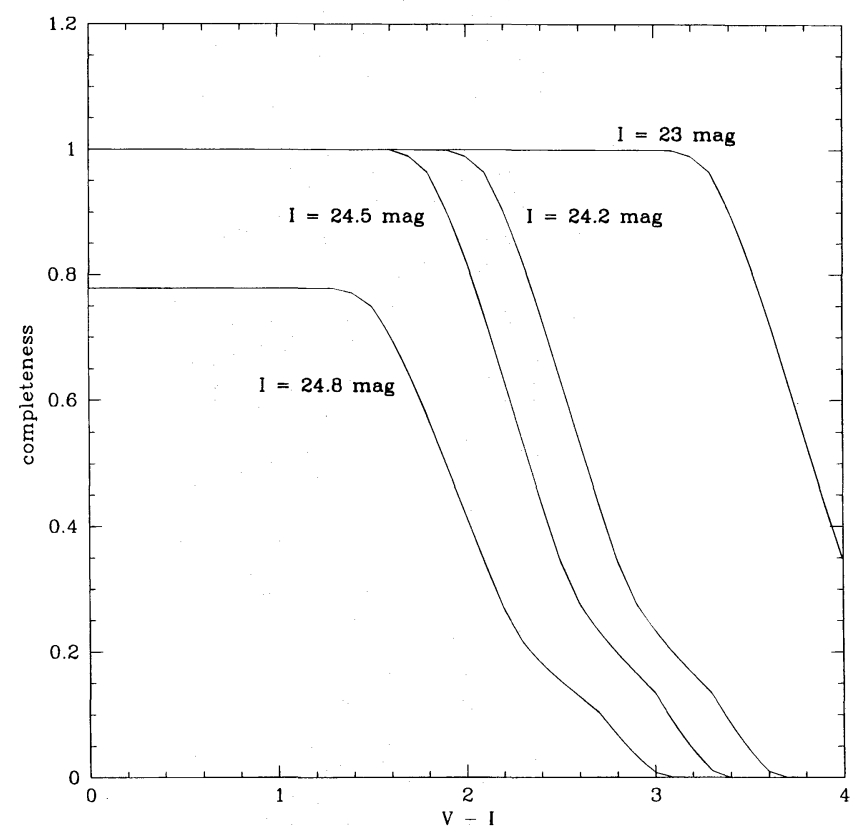

FIG. 5.-Completeness level of our sample as a function of $V-I$, for different values of $I$; the ADDSTAR algorithm was used to determine these curves. we shall argue in $\S 5.2$ : some of the points plotted in Figures 3 and 4 may simply be spurious because of the crowding of our field, but we estimate photometric errors cannot account for all of them.

The solid lines superimposed on the $(I, V-I)$ colormagnitude diagram are the giant branch loci of six wellstudied galactic globular clusters spanning various metal abundances (see Table 2). In order to place those curves on a common scale with the NGC 5128 RGB stars, we need to know the reddenings and the distance moduli of both NGC 5128 and the globular clusters; for the latter, the parameters adopted here are those found in Table IX of Da Costa \& Armandroff (1990). The reddening for NGC 5128 has been taken from Burstein \& Heiles (1984), using the $E(V-I) /$ $E(B-V)$ relation of Dean, Warren, \& Cousins (1978); this gives $E(V-I)=0.16 \pm 0.02 \mathrm{mag}$ and $A_{I}=0.22 \pm 0.02$ mag. We tentatively adopt a distance modulus $(m-M)_{0}=$ $27.72 \mathrm{mag}$ using the results discussed in Hui et al. (1993); this value is in agreement with both the distance derived

TABLE 2

Galactic Globular Clusters Adopted FOR A COMPARISON WITH NGC 5128

\begin{tabular}{ccc}
\hline \hline Cluster & {$[\mathrm{Fe} / \mathrm{H}]$} & $M_{I, \text { IRGB }}$ \\
\hline NGC $7078(\mathrm{M} 15) \ldots \ldots \ldots$ & -2.17 & -4.095 \\
NGC $6397 \ldots \ldots \ldots \ldots \ldots$ & -1.91 & -3.765 \\
NGC $7089(\mathrm{M} 2) \ldots \ldots \ldots$ & -1.58 & -4.035 \\
NGC $6752 \ldots \ldots \ldots \ldots \ldots$ & -1.54 & -3.948 \\
NGC $1851 \ldots \ldots \ldots \ldots \ldots$ & -1.29 & -4.021 \\
NGC $104(47$ Tuc $) \ldots \ldots$ & -0.71 & -4.039
\end{tabular}


from the planetary nebulae luminosity function and the one inferred from the surface brightness fluctuation technique. From inspection of Figure 3 it appears that with the $\therefore$ adopted modulus the tip of the RGB in NGC 5128 has 1he same luminosity as the TRGB in the globular clusters; in other words, the distance of NGC 5128 determined by comparing the luminosity of the RGB tips (see $\S 5.1)$ is consistent with the distance derived with the other two (independent) techniques.

Another relevant piece of information that can be inferred from a comparison of the RGBs in NGC 5128 and in the globular clusters is the metallicity distribution, which will be discussed in $\S 6$. For a given luminosity, metal-rich stars are redder; the relation between color and metallicity is nonlinear.

\section{LUMINOSITY FUNCTION}

\subsection{The RGB Tip}

The RGB tip luminosity is a reliable distance indicator because its $I$ magnitude depends very little upon age (see Green, Demarque, \& King 1987) and metallicity, at least for abundances equal to or less than $[\mathrm{Fe} / \mathrm{H}]=-0.7$ dex (see Da Costa \& Armandroff 1990, hereafter DA90). ${ }^{12}$ From the giant branch loci in the $\left[M_{I},(V-I)_{0}\right]$ plane derived by DA90, we adopted an absolute magnitude $M_{I}=-3.98$ $\pm 0.12 \mathrm{mag}$ for the tip; hence, the distance modulus of a galaxy or a cluster of stars can be expressed by the simple relation

$$
(m-M)_{0}=I_{\mathrm{TRGB}}-(-3.98)-A_{I} .
$$

In our case, we know that $A_{I}=0.22 \pm 0.02 \mathrm{mag}$, and we need only to determine the tip of the RGB from the luminosity function (LF). It is possible in some cases to estimate the position of the discontinuity in the luminosity function simply by eye, but for distant galaxies like NGC 5128, large photometric errors tend to smear out the edge; in a more quantitative approach, one can differentiate the luminosity function and associate the tip of the RGB with the point at which the first derivative has a maximum (the point of steepest ascent in the LF). The uncertainty in the detection of the edge depends mostly upon the size of the bins into which the data can be collected: in our case we cannot choose bins smaller than $0.1 \mathrm{mag}$ in the LF owing to the photometric error. A comprehensive analysis of this method with a computer simulation using artificial LFs can be found in Lee, Freedman, \& Madore (1993b), Madore \& Freedman (1995), and Sakai, Madore, \& Freedman (1996). The solid line in Figure 6 displays the LF of NGC 5128; only the stars with $V-I>1.5$ mag have been considered, in order to make the detection of the edge a little easier; the first derivative of the LF is shown in Figure 8 (solid line). Five-point smoothing was applied to the luminosity function derivative with the first and last points in the "boxcar" given half-weight; three-point smoothing produced similar results.

\footnotetext{
12 The DA90 giant branches show that for $[\mathrm{Fe} / \mathrm{H}]>-0.7$ the giant branch tip is fainter than specified here. This is due to the effects of bolometric correction in cool stars. (Their luminosities are predicted to be similar to or greater than those of less metal-rich stars). These effects do not bias the RGB tip distance indicator provided metal-poor stars are in fact present in the stellar population. They simply soften the luminosity function discontinuity.
}

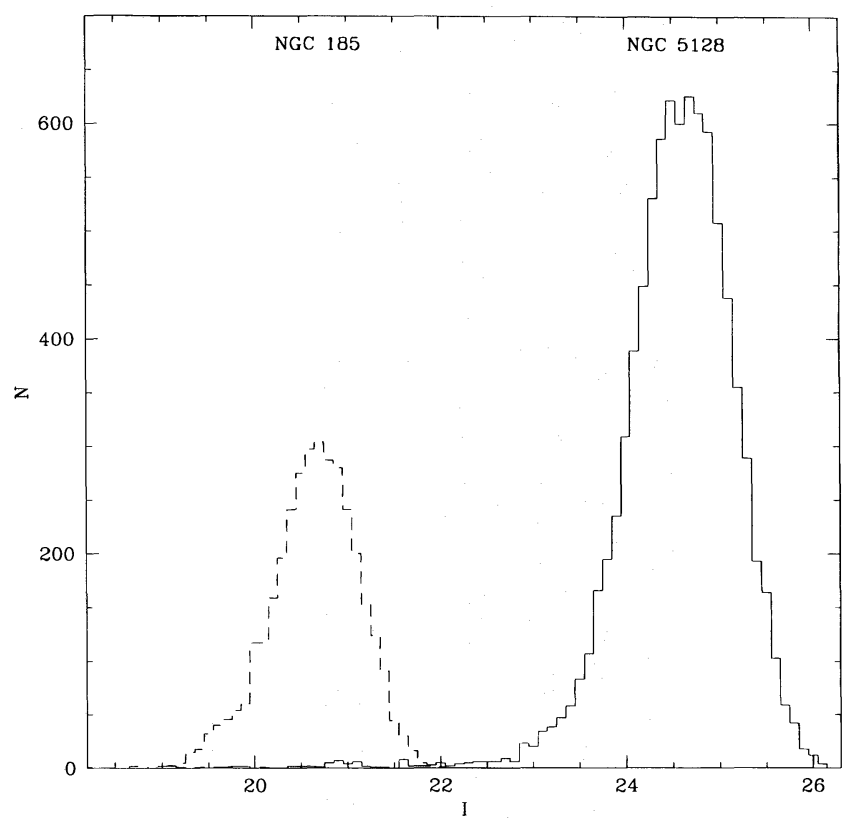

FIG. 6. - I luminosity functions for the RGB stars in NGC 5128 (solid line) and NGC 185 (dashed line); only stars redder than $V-I=1.5$ have been considered.

The result we obtained is $I_{\mathrm{TRGB}}=24.1 \pm 0.1 \mathrm{mag}$ : this gives a true distance modulus $(m-M)_{0}=27.86 \pm 0.16$ mag, in agreement with the modulus determined from the planetary nebulae luminosity function, $(m-M)_{0}=27.73$ $\pm 0.14 \mathrm{mag}$, and with that derived using the surface brightness fluctuation technique, $(m-M)_{0}=27.71 \pm 0.10 \mathrm{mag}$ (Hui et al. 1993). This confirms that the most likely distance to the galaxy is $3.7 \pm 0.2 \mathrm{Mpc}$. The value $I_{\mathrm{TRGB}}=24.0$ $\pm 0.1 \mathrm{mag}$ is obtained by considering only the stars detected in PC1 (see Fig. 7): they are a small fraction $(1 / 20)$ of the total number, but $\mathrm{PC} 1$ has a better resolution $\left(0^{\prime \prime} 046\right.$

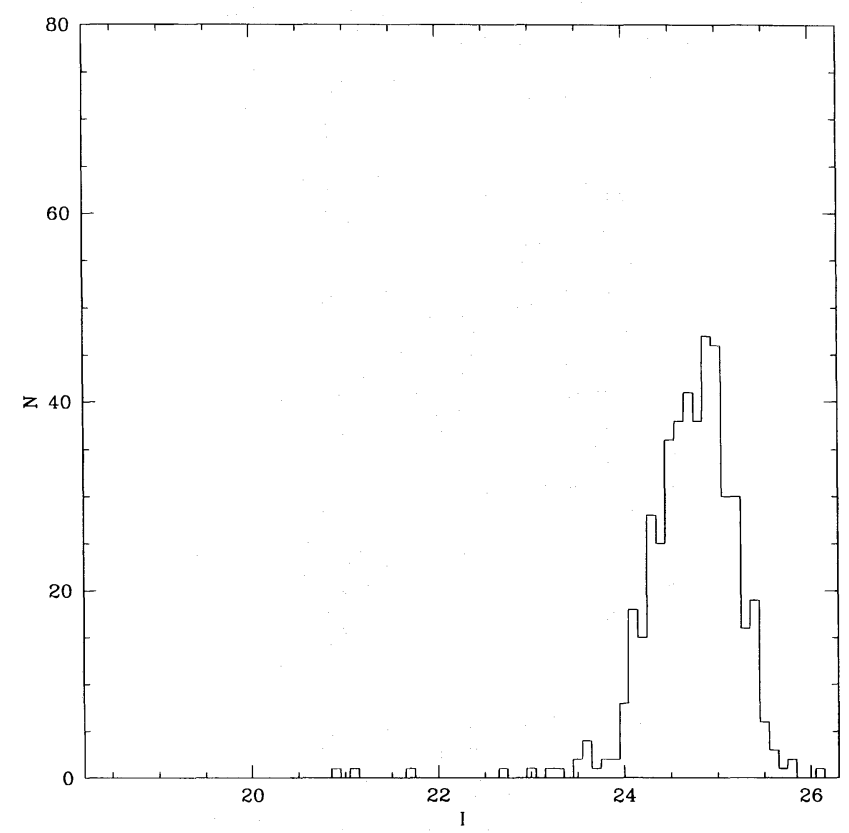

FIG. 7.-I luminosity functions for the RGB stars in NGC 5128 detected in PC1; only stars redder than $V-I=1.5$ have been considered, as before. The turn-up in the $\mathrm{LF}$ at $I=24.0 \pm 0.1 \mathrm{mag}$ is more evident. 
pixel ${ }^{-1}$, while it is 0.1 pixel $^{-1}$ in the Wide Field chips); if we assume this value to be more reliable, the modulus will be $(m-M)_{0}=27.76 \pm 0.16 \mathrm{mag}(3.6 \pm 0.2 \mathrm{Mpc})$.

As a test of the method used to detect the discontinuity in the LF at the tip of the RGB we repeated the same analysis on the LF of the dwarf elliptical galaxy NGC 185, for which the distance modulus had been determined with independent techniques (Lee et al. 1993a; Lee 1995). The true distance modulus of NGC 5128 is now given by

$$
\begin{aligned}
(m-M)_{0, \mathrm{NGC} 5128}= & (m-M)_{0, \mathrm{NGC} 185} \\
& +I_{\mathrm{TRGB}, \mathrm{NGC} 5128}-I_{\mathrm{TRGB}, \mathrm{NGC} 185} \\
& +A_{I, \mathrm{NGC} 185}-A_{I, \mathrm{NGC} 5128} .
\end{aligned}
$$

The relative shift between the tip of the RGB in the two galaxies appears to be $\Delta I_{\mathrm{TRGB}}=3.65 \pm 0.15 \mathrm{mag}$, the tip being found at $I=20.35 \pm 0.10$ mag for NGC 185 (in good agreement with the value of 20.30 chosen by Lee). We then assume $A_{I, \mathrm{NGC} 185}=0.38 \mathrm{mag}$ and adopt for NGC 185 the distance modulus derived from the absolute $V$ magnitudes of 151 RR Lyrae stars (Saha \& Hoessel 1990): $(m-M)_{0, \mathrm{NGC}} 185=24.02 \pm 0.25 \mathrm{mag}$; this leads to a distance modulus $(m-M)_{0, \mathrm{NGC}} 5128=27.8 \pm 0.3 \mathrm{mag}$. The first derivative of the LF for NGC 185 is shown in Figure 8 as a dashed line.

A second galaxy to which we can relate the RGB tip in NGC 5128 is M31 where Davidge (1993) has found the discontinuity in the $I$-band luminosity function at $I=20.6 \pm 0.1$ mag. Assuming $(m-M)_{0}=24.3$ and

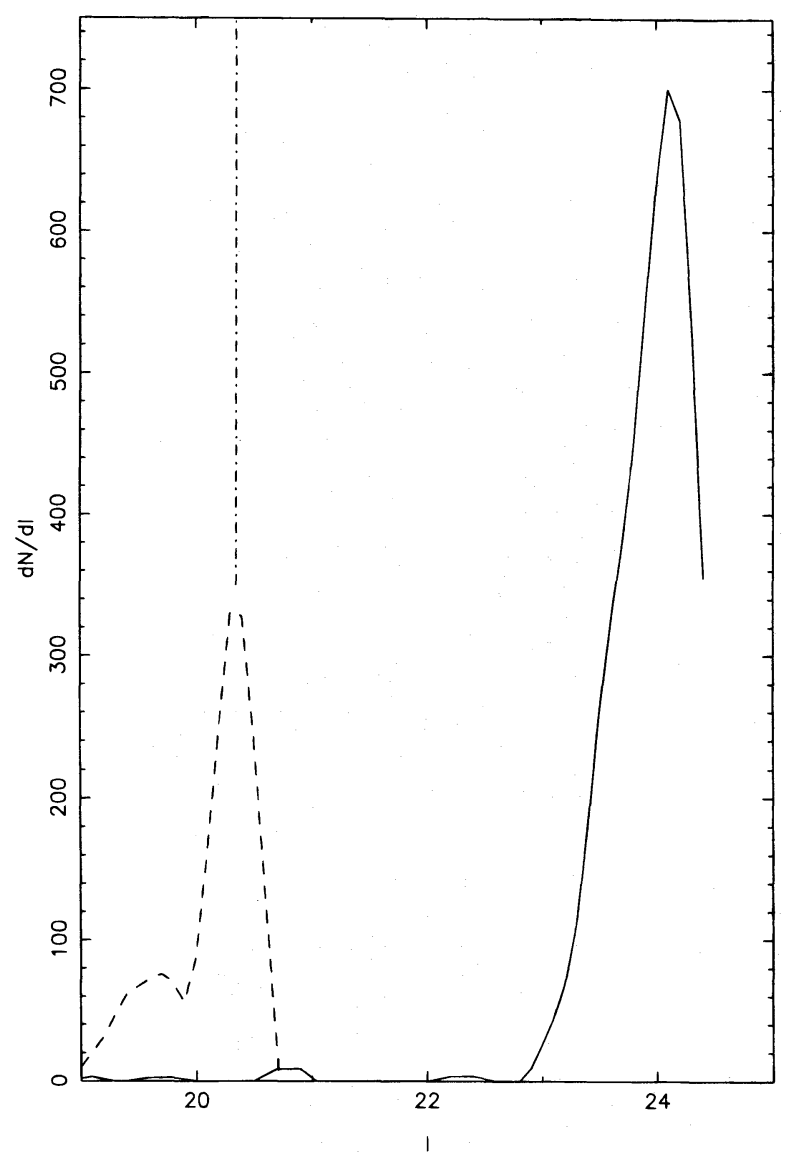

FIG. 8.-First derivative of the $I$ luminosity function in NGC 5128 (solid line) and NGC 185; we can associate the tip of the RGB with the maximum in the LF's first derivative.
$E(B-V)=0.08$ for $\mathrm{M} 31$ as Davidge did, we obtain $(m-M)_{0}=27.63 \pm 0.2$ for NGC 5128 from the PC measurement of the RGB tip. Davidge's CMD bears a strong resemblance to that of NGC 5128.

These results are consistent with the distance modulus from surface brightness fluctuations (Tonry \& Schechter 1990). With Tonry's (1995) calibration, $(m-M)_{I}=27.9$ \pm 0.08 and $(m-M)_{0}=27.68$. SN 1986G also yields consistent distances, but its large reddening vitiates its use as a standard candle (Frogel et al. 1987; Phillips 1993).

\subsection{Stars above the RGB Tip}

Although the number density of stars falls quickly for magnitudes $I<I_{\text {TRGB }}$ as expected, a few stars are still detected at higher luminosities: we found about 150 stars with $I<23 \mathrm{mag}$ and about 50 for $I<22 \mathrm{mag}$, as shown in Figure 13 and in the C-M diagrams of Figures 3 and 4 . We can think of four possible explanations for these stars:

1. They are simply blends of two or more normal red giant branch stars: in a crowded field two or more stars may happen to be overlapping and may be classified as one bright star by ALLFRAME. We tested this possibility by adding to our images some 1000 artificial stars fainter than $I=24$ mag and by checking out how many of them were eventually assigned a magnitude brighter than $I=23 \mathrm{mag}$ by ALLFRAME. We conclude that photometric errors can explain between $30 \%$ and $50 \%$ of the detections $0.5 \mathrm{mag}$ above the TRGB magnitude limit.

2. They are foreground Galactic stars. Using the star density calculated by Reid (1995), we estimate that field contamination in our $5.8 \mathrm{arcmin}^{2}$ field can account for up to $20-25$ stars with $18<I<24 \mathrm{mag}$ and $0<V-I<4$; in particular, at most, two or three of them are expected to be found with $22<I<23.5 \mathrm{mag}$ and $0<V-I<4$. Therefore, the effect of Galactic contamination can be neglected at these luminosities, while they can probably explain all the stars seen at $I<22$ mag.

3 . We are detecting real NGC 5128 stars other than lowmass giant branch stars. The presence of a few bright, blue stars with $I \gtrsim 23$ mag and $(V-I) \lessgtr 1$ is consistent with the stellar distribution in the C-M diagrams of NGC 185; Lee et al. (1993a) suggests these are young, Population I stars, but it seems to us their status is not understood. One might compare them with similar bright stars observed in the stellar constellation Shapley III in the LMC (see Reid, Mould, \& Thompson 1987); in fact, stars above the RGB tip in NGC 5128 appear to be generally similar to those in the LMC. But in the absence of $\mathbf{H}$ I gas or interstellar material on the south side of the halo of NGC 5128 the analogy seems strained.

In Figures 9 and 10 we approximately identified the position on the color-magnitude diagrams of the different classes of stars discussed above.

4. They are upper asymptotic giant branch stars.

The majority of these stars appear to be upper asymptotic giant branch (AGB) stars of the kind that are seen prominently in the nuclear bulge of the Milky Way and in the LMC (Frogel et al. 1990b; Frogel, Mould, \& Blanco 1990a). We can infer something more about the AGB stars in NGC 5128 by plotting an $\left[M_{\text {bol }}-(V-I)_{0}\right]$ colormagnitude diagram, as we did in Figure 11; we adopted a distance modulus $(m-M)_{0}=27.72 \mathrm{mag}$, while the bolometric correction to the I magnitude was taken from 


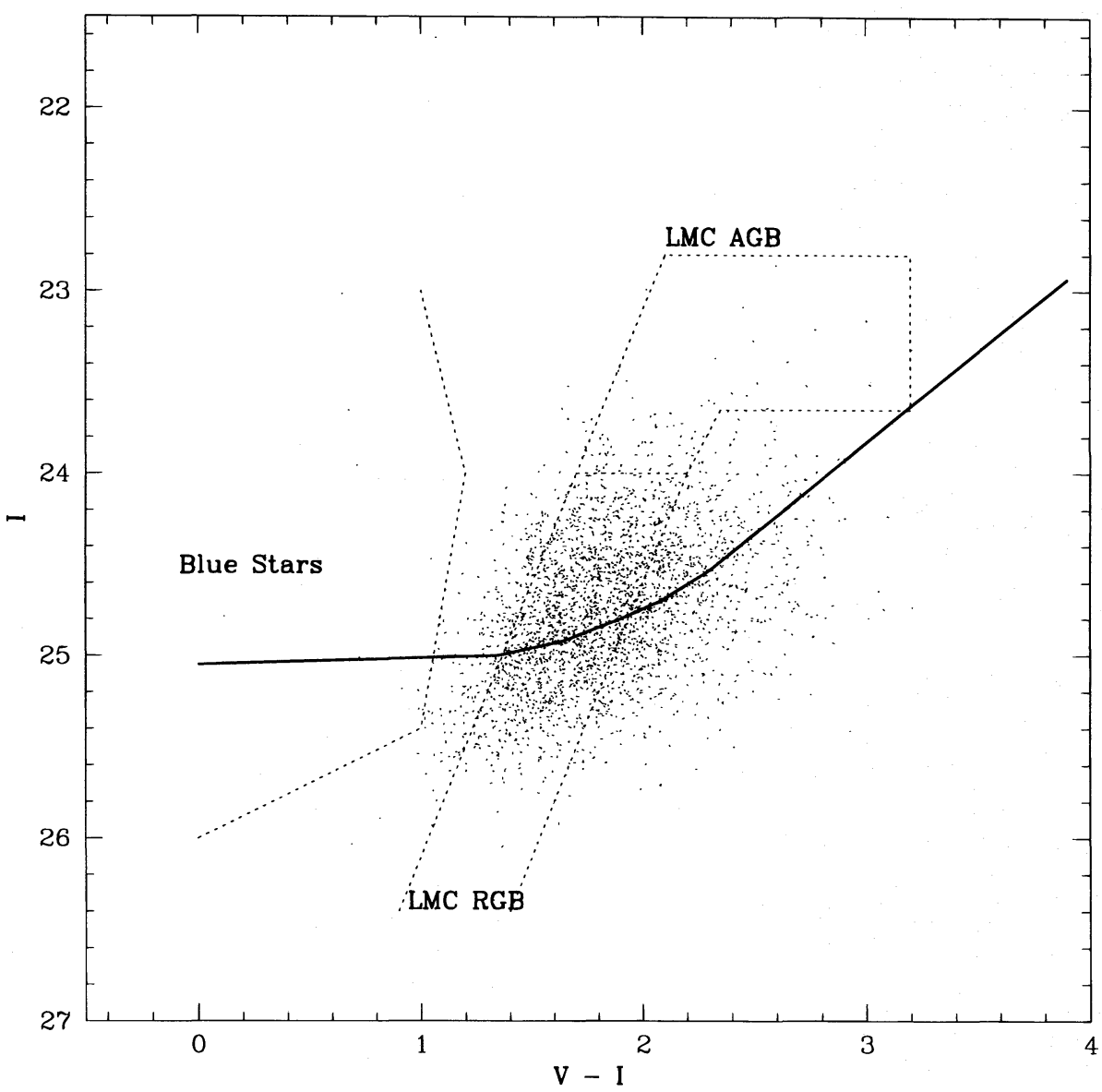

Fig. 9.-Color-magnitude diagram in the $(I, V-I)$ plane, with the $50 \%$ completeness level indicated by a solid line; the dotted lines defines the approximate locus of the blue tars (see text) and the locus of the RGB and AGB stars in the Large Magellanic Cloud field studied by Reid et al. (1987).

Da Costa \& Armandroff 1990 as

$$
\mathrm{BC}_{I}=0.881-0.243(V-I)_{0}=0.920-0.243(V-I) \text {. }
$$

From this diagram, and from the slope of the bolometric luminosity function plotted in Figure 12, we see that the AGB extends up to $M_{\text {bol }} \sim-5$ mag. A natural explanation of the $\sim 300$ stars in the range $(-5,-4)$ in $M_{\text {bol }}$ is that a very significant fraction of the star formation in NGC 5128 occurred just $\sim 5$ Gyr ago. Aaronson \& Mould (1985) have shown that LMC clusters of age $5 \mathrm{Gyr}$ reach $M_{\mathrm{bol}}=-4.5$ on the AGB. Allowing for the possibility that some half of the stars in this magnitude range may have been promoted there by confusion, we see that the ratio of these stars to stars at the RGB tip is similar in NGC 5128 to its value in M32 (Freedman 1989, 1992). O'Connell $(1980,1986)$ has argued convincingly that the age of the dominant stellar population in M32 is 5 Gyr (but see Renzini 1992). Although the current star formation in NGC 5128 is no doubt of much more recent origin, if the elliptical galaxy NGC 5128 formed in a merger, could this have been its approximate date?

We can calculate the number, $n$, of upper AGB stars expected in a $5 \mathrm{Gyr}$ old population with the aid of

$$
n=b L_{V} t
$$

(Renzini \& Buzzoni 1986), where $L_{V}$ is the visual luminosity of the population and $t$ is the lifetime of stars in this interval on the AGB. The luminosity sampled can be estimated from the surface photometry of van den Bergh (1976). Assuming the average surface brightness is $\mu_{V}=23.5 \mathrm{mag} \operatorname{arcsec}^{-2}$, we obtain $L_{V}=7 \times 10^{7} L_{\odot}$ for the area sampled. Adopting $b=3 \times 10^{-11}$ and $t=0.7 \times 10^{6} \mathrm{yr}$ for the half-magnitude between -4 and -4.5 in $M_{\text {bol }}$ (Iben, Renzini, \& Schramm 1978), one predicts 1500 stars. This would constrain a burst of star formation from a merger to add only $\sim 10 \%$ to the pre-existing stellar population.

Moreover, we cannot rule out the hypothesis that these upper AGB stars represent a high-metallicity tail of the distribution, which in some way, possibly a low mass-loss rate, ascend the AGB like a younger population. Stars with $M_{\text {bol }}=-5$ are observed in the nuclear bulge of the Milky Way (Terndrup, Frogel, \& Whitford 1990), although these stars may themselves be of intermediate age (Holtzman et al. 1992).

In summary, the quantitative evidence is against the hypothesis that the halo of Cen A is a simple stellar population of age $\sim 5$ Gyr. Rather, AGB star counts seem to push the major epoch of star formation in NGC 5128 back earlier than $5 \mathrm{Gyr}$ ago, although unknown mass-loss/ metallicity dependences allow a great deal of flexibility to the model maker.

\section{METALLICITY DISTRIBUTION}

The color dispersion in the magnitude below the RGB tip is large and real and is naturally explained by a range in metallicity in this halo sample. At $I=24.4 \mathrm{mag}$, the color dispersion is $\sigma \simeq 0.54 \mathrm{mag}$; the measuring error in color is $\sigma \simeq 0.15 \mathrm{mag}$. 


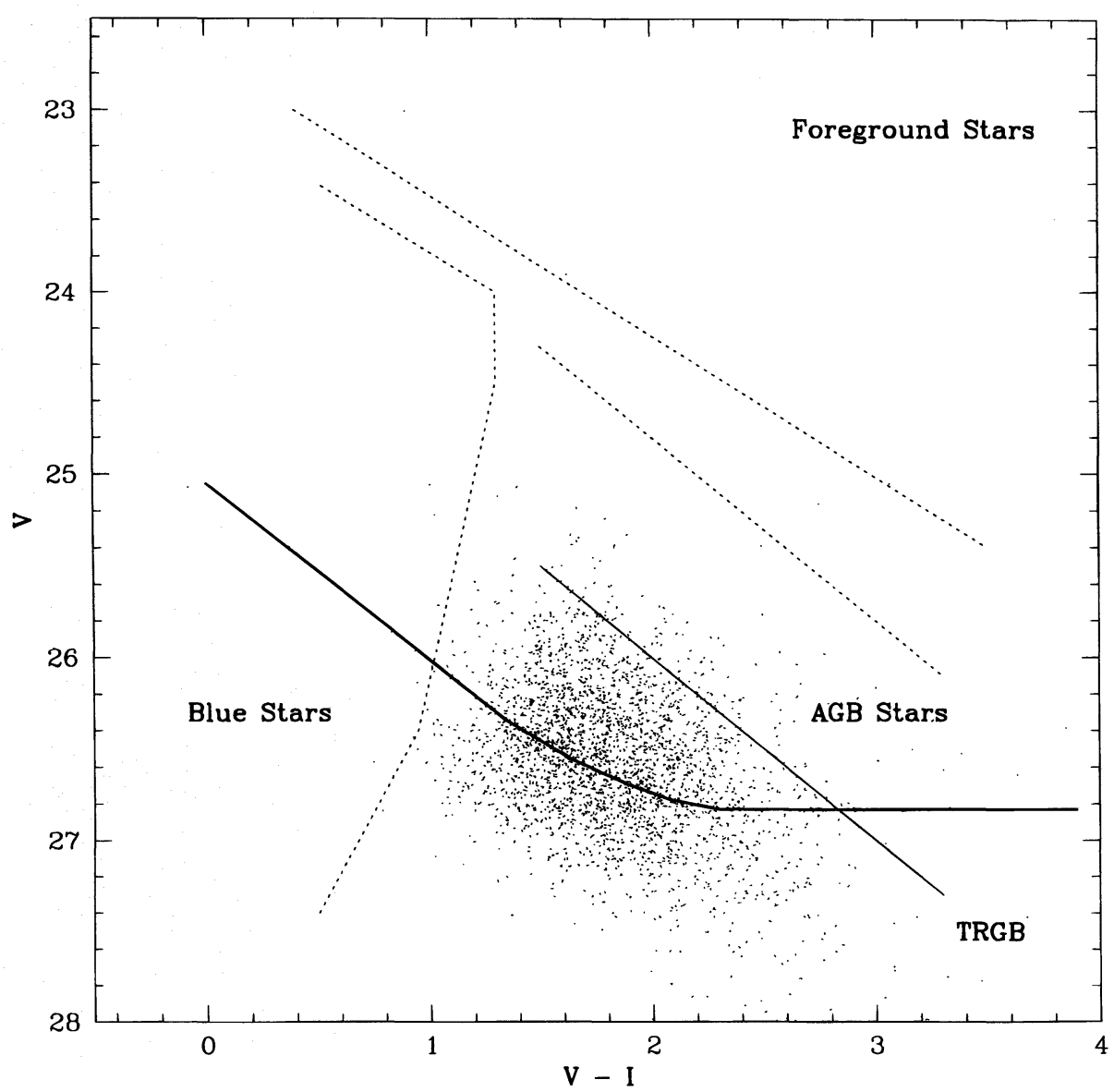

FIG. 10.-Color-magnitude diagram in the $(V, V-I)$ plane; again, we have displayed the loci of the blue stars and of the AGB stars; the bright, red stars in the upper right-hand corner are likely to be Galactic foreground stars; the thin, solid line marks the tip of the RGB, and the thick line is the $50 \%$ completeness level of our sample.

The relation between the intrinsic color of the RGB and the metal content of its stars can be approximated by (see Da Costa \& Armandroff 1990)

$$
[\mathrm{Fe} / \mathrm{H}]=-15.16+17.0(V-I)_{0,-3}-4.9\left[(V-I)_{0,-3}\right]^{2} \text {, }
$$

where $(V-I)_{0,-3}$ is the color of the RGB at $M_{I}=-3$, i.e., 1 mag below the RGB tip. The relation is valid for $-2.2<[\mathrm{Fe} / \mathrm{H}]<-0.7$. Alternatively, for the same range of metal abundances, we can adopt the relation

$$
\begin{aligned}
{[\mathrm{Fe} / \mathrm{H}]=} & -12.64+12.61(V-I)_{0,-3.5} \\
& -3.33\left[(V-I)_{0,-3.5}\right]^{2},
\end{aligned}
$$

derived by Lee et al. (1993a), where we defined $(V-I)_{0,-3.5}$ as the intrinsic color of the RGB one-half mag below the tip; this relation is more useful in our case, because of the low level of completeness affecting our data at $I \simeq 25 \mathrm{mag}$.

In Figure 13 we show horizontal cuts across the RGB at three such magnitudes after correction for completeness. These distribution functions in turn constrain the metallicity distribution function $d n / d[\mathrm{Fe} / \mathrm{H}]$. We find that over the range $(-1.5,-0.5)$ in $[\mathrm{Fe} / \mathrm{H}] d n / d[\mathrm{Fe} / \mathrm{H}]$ is a rising function, although only for $I=24.2$ do we have a sample of stars with $[\mathrm{Fe} / \mathrm{H}]>-0.7$ and completeness $>50 \%$. We conclude that the mean value of $[\mathrm{Fe} / \mathrm{H}]$ exceeds -0.9 in this halo field in NGC 5128, conservatively disregarding samples at $I=24.5$ and 24.8 that require mean values exceeding -0.75 , but rely on completeness corrections of a factor of 2 or more.

We note that Mould \& Kristian (1986) using similar techniques found $[\mathrm{Fe} / \mathrm{H}]>-0.6$ for their halo field in M31. If the halo metallicity/luminosity relation is a guide ( $\mathrm{Da}$ Costa $\&$ Mould 1988), these relatively high metallicity values may be characteristic of the halos of luminous galaxies with $M_{V} \simeq-21$. The similarity of the NGC 5128 and M31 halo red giant branches (Davidge 1993) is particularly striking.

\section{CONCLUSIONS}

The main conclusions we can derive from the present work are as follows:

1. The distance modulus for NGC 5128 inferred from the luminosity of the tip of the red giant branch is in agreement with the values previously determined using the planetary nebulae luminosity function and surface brightness fluctuations; for NGC 185 and M31, it is also in agreement with the distance derived from the luminosity of RR Lyrae stars. This is a further indication of the reliability of the TRGB method, extensively discussed in Madore \& Freedman (1995).

2. We notice the presence of bright stars, bluer than $(V-I)=1$; bright, blue stars have also been observed in the dwarf elliptical NGC 185 by Lee et al. (1993a).

3 . On the other hand, the observed number of AGB stars in the range $-5<M_{\mathrm{bol}}<-4$ mag (well above the RGB tip) constrains an episode of star formation in consequence 


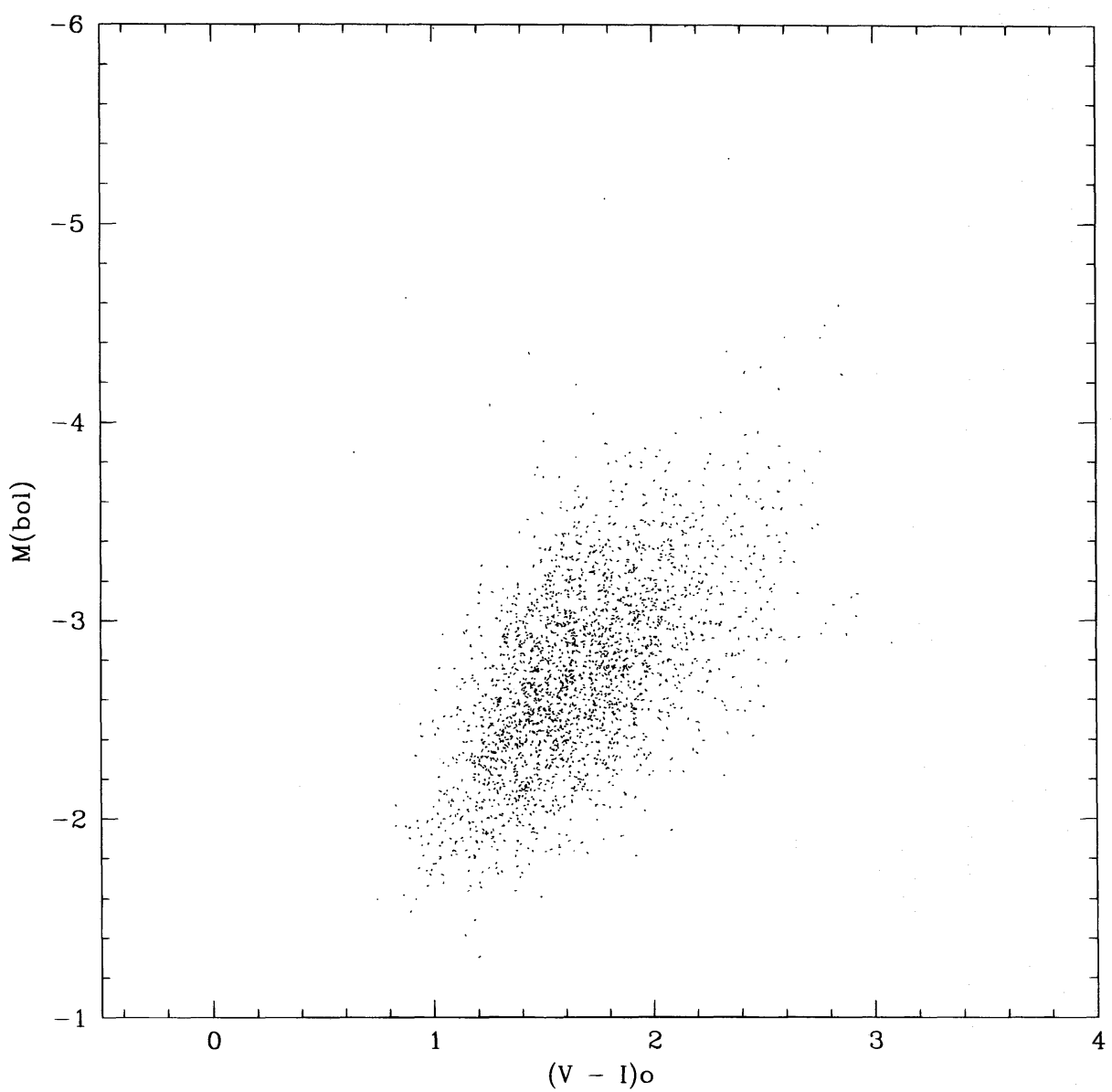

FIG. 11.-Color-magnitude diagram in the $\left[M_{\text {bol }},(V-I)_{0}\right]$ plane for the stars in NGC 5128

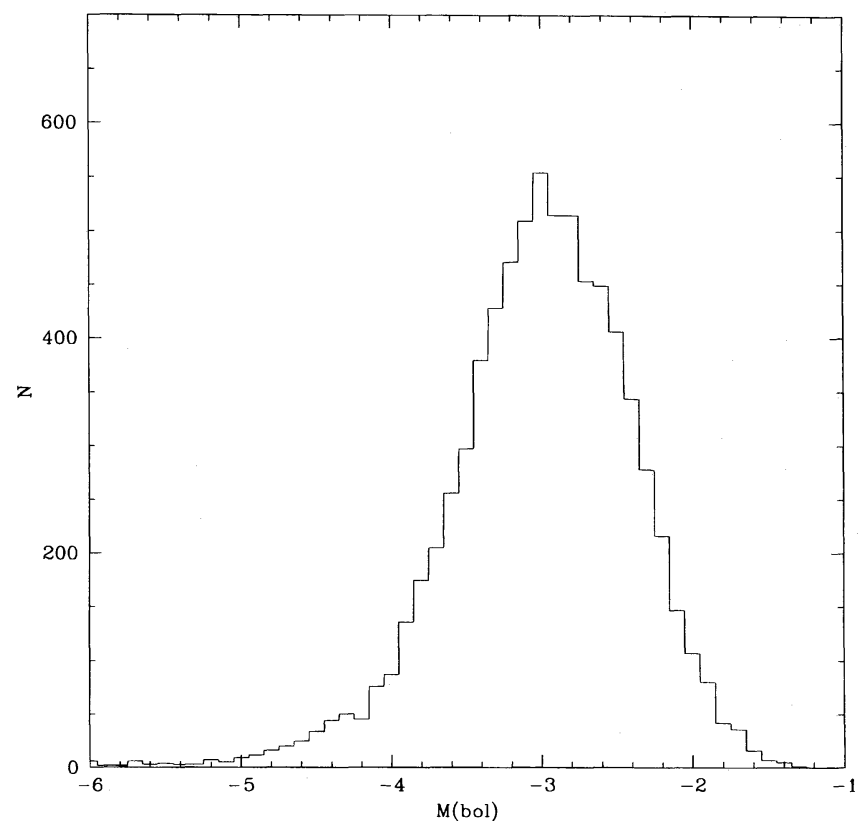

FIG. 12. $-M_{\text {bol }}$ luminosity function for our field in the halo of NGC 5128 ; the brightest AGB stars extend up to $M_{\text {bol }} \sim-5$. of a merger to add less than about $10 \%$ to the pre-existing stellar population; the major epoch of star formation was probably earlier than $5 \mathrm{Gyr}$ ago, although large uncertainties remain in the evolutionary models.

4. Finally, the position of the red giant branch in the $\mathrm{C}-\mathrm{M}$ diagram implies a mean value of $[\mathrm{Fe} / \mathrm{H}]$ higher than -0.9 (considerably larger than the mean metallicity in NGC 185); relatively high metallicity values have previously been found in a halo field in M31. The crowding in our field (causing large photometric error) and, above all, a completeness level falling below the $50 \%$ limit 1 mag below the tip of the RGB are the main sources of uncertainty in our analysis.

We are grateful to Daya Rawson and Peter Stetson for contributing their expertise in photometry and Neill Reid for star count calculations. We thank Gary Da Costa and Carl Grillmair for critical reading of the manuscript and John Graham and Jay Frogel for their stimulating remarks and suggestions. This research was carried out by the WFPC2 Investigation Definition Team for JPL and was sponsored by NASA through contract NAS7-1260. 

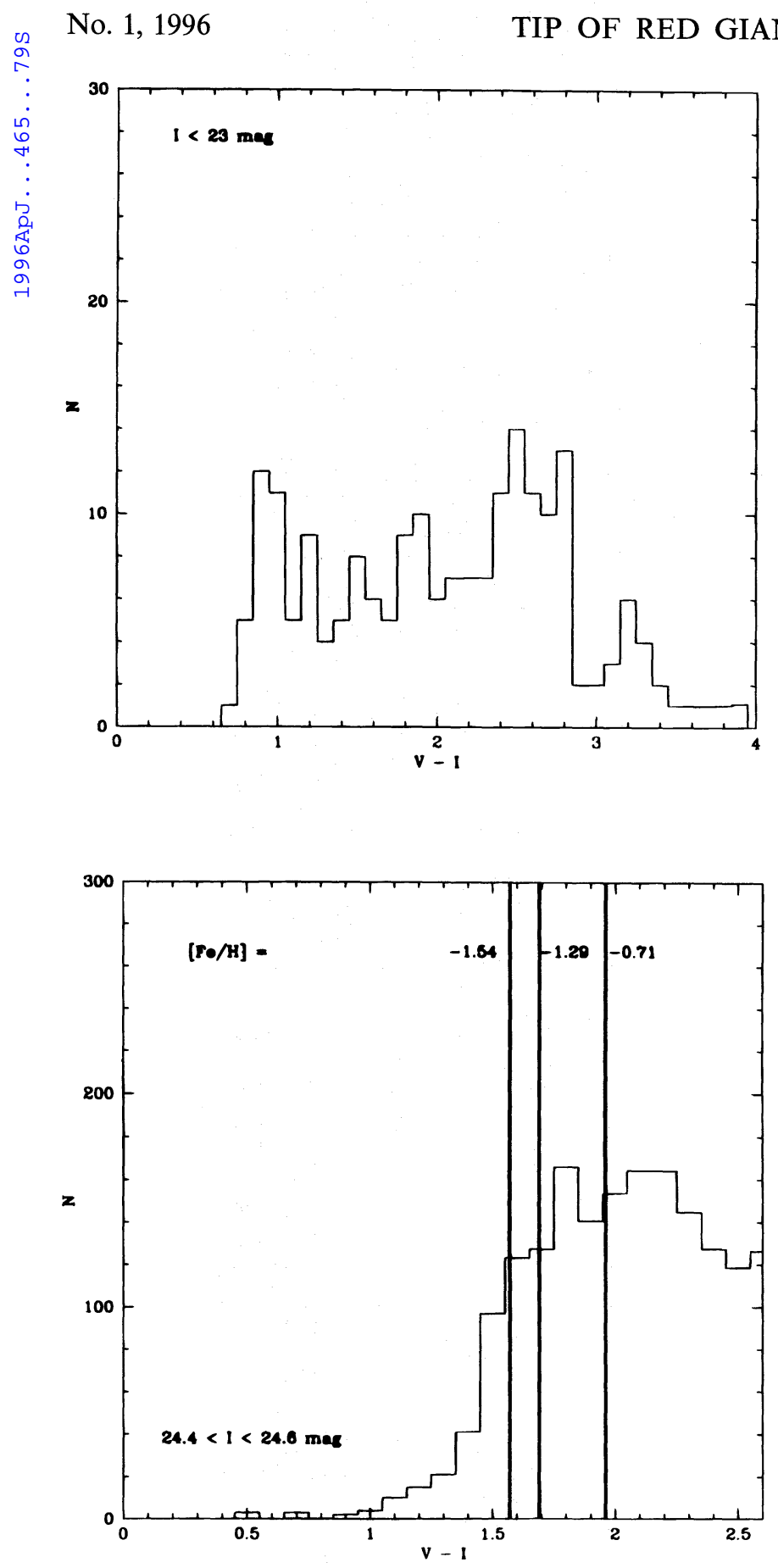

FIG. 13.-Color distribution of the stars in the NGC 5128 field at different values of $I$. Part of the detections at $I<23$ mag are due to AGB stars and Galactic foreground stars; about half of them are simply due to photometric errors caused by the overlapping of two or more stars in our crowded field. The locus of the RGB in three globular clusters with different metallicities has also been displayed; the clusters are NGC $6752([\mathrm{Fe} / \mathrm{H}]=-1.54)$, NGC 1851 $([\mathrm{Fe} / \mathrm{H}]=-1.29)$, and $47 \mathrm{Tuc}([\mathrm{Fe} / \mathrm{H}]=-0.71)$.

\section{REFERENCES}

Aaronson, M., \& Mould, J. 1985, ApJ, 288, 551

Burstein, D., \& Heiles, C. 1984, ApJS, 54, 33

Burrows, C. J., ed. 1994, Wide Field and Planetary Camera 2 Instrument Handbook (Baltimore: STScI)

Da Costa, G. S., \& Armandroff, T. E. 1990, AJ, 100, 162 (DA90)

Davidge, T. 1993, ApJ, 409, 191

Dean, J. F., Warren, P. R., \& Cousins, A. W. J. 1978, MNRAS, 183, 569

de Vaucouleurs, G. 1993, ApJ, 415, 33

Freedman, W. 1989, AJ, 98, 1285.

. 1992, in IAU Symp. 149, The Stellar Populations of Galaxies, ed. B. Barbuy \& A. Renzini (Dordrecht: Kluwer), 169

Frogel, J. A., Gregory, B., Kawara, K., Laney, D., Phillips, M. M.,

Terndrup, D., Vrba, F., \& Whitford, A. E. 1987, ApJ , 315, L129

Frogel, J., Mould, J., \& Blanco, V. 1990a, ApJ, 352, 96
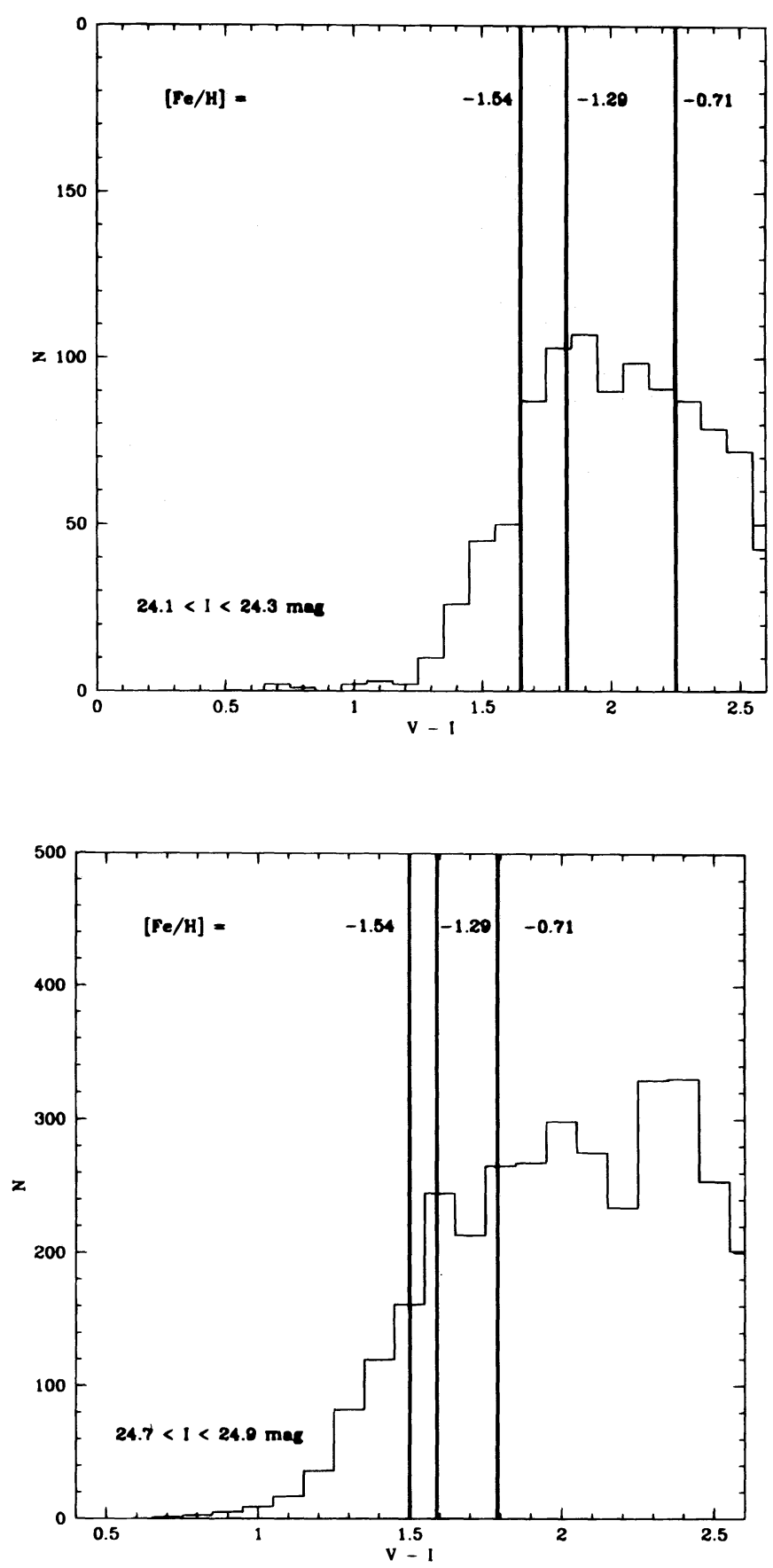

Frogel, J., Terndrup, D., Blanco, V., \& Whitford, A. 1990b, ApJ, 353, 494 Graham, J. 1979, ApJ, 232, 60

Green, E. M., Demarque, P., \& King, C. R. 1987, The Revised Yale Isochrones and Luminosity Functions (New Haven: Yale Univ. Obs.)

Holtzman, J. A., Burrows, C. J., Casertano, S., Hester, J. J., Trauger, J. T., Watson, A. M., \& Worthey, G. 1995a, PASP, 107, 1065

Holtzman, J. A., et al. 1992, AJ, 103, 691

Holtzman, J. A., Hester, J. J., Casertano, S., Trauger, J. T., Watson, A. M., \& WFPC-2 IDT. 1995b, PASP, 107, 156

Hui, X., Ford, H. C., Ciardullo, R., \& Jacoby, G. H. 1993, ApJ, 414, 463

Iben, I., Renzini, A., \& Schramm, D. N. 1978, in Advanced Stages in Stellar

Evolution, ed. P. Bouvier \& A. Maeder (Geneva: Geveva Obs.), 170

Lee, M. G. 1995, private communication 


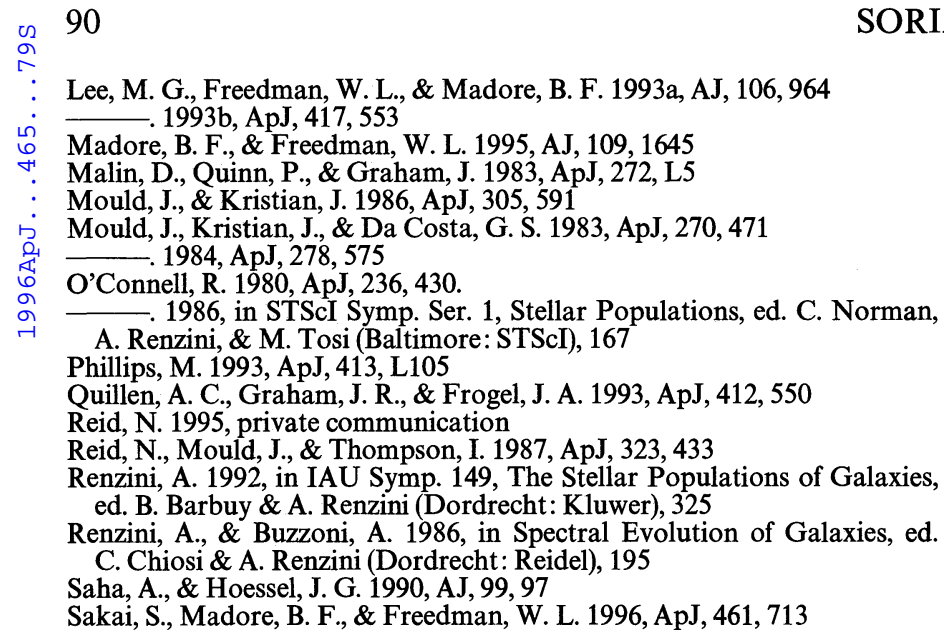

Renzini, A., \& Buzzoni, A. 1986, in Spectral Evolution of Galaxies, ed. C. Chiosi \& A. Renzini (Dordrecht: Reidel), 195

Saha, A., \& Hoessel, J. G. 1990, AJ, 99, 97

Sakai, S., Madore, B. F., \& Freedman, W. L. 1996, ApJ, 461, 713

SORIA ET AL.

Schiminovich, D., van Gorkom, J. H., van der Hulst, J. M., \& Kasow, S. 1994, ApJ, 423, L101

Schweizer, F. 1994, in IAU Symp. 164, Stellar Populations, ed. P. van der Kruit (Dordrecht: Kluwer), 275

Stetson, P. B. 1991a, in 3rd ESO/ST-ECF Data Analysis Workshop, ESO Conference \& Workshop Proc. 38, ed. P. J. Grosbol \& R. H. Warmels (Garching: ESO), 187

- 1991b, in ASP Conf. Proc. 25, Astronomical Data Analysis Software and Systems. I, ed. D. Worrall, C. Biemesderfer, \& J. Barnes (San Francisco: ASP), 297 - 1994, PASP, 106, 250 1995, private communicatio

Sutherland, R., Bicknell, G. V., \& Dopita, M. A. 1993, ApJ, 414, 510

Terndrup, D., Frogel, J. A., \& Whitford, A. E. 1990, ApJ, 357, 453

Tonry, J. 1995, Heron Island Workshop (http:// qso.lanl.gov/ heron)

Tonry, J., \& Schechter, P. 1990, AJ, 100, 1794

Toomre, A., \& Toomre, J. 1972, ApJ, 178, 623

Trauger, J. T., et al. 1994, ApJ, 435, L3

van den Bergh, S. 1976, ApJ, 208, 673 